\title{
Prevención del tabaquismo activo y pasivo en la infancia
}

\author{
J. Galbe Sánchez-Ventura ${ }^{a}$, R. Córdoba García ${ }^{b}$, N. García Sánchez ${ }^{c}$ \\ aPediatra. CS Torrero-La Paz. Zaragoza. España. \\ ${ }^{b}$ Medico de Familia. CS Delicias Sur. Zaragoza. España. \\ 'Pediatra. CS Delicias Sur. Zaragoza. España.
}

Rev Pediatr Aten Primaria. 2009; I I (Supl I 7):s359-s369

José Galbe Sánchez-Ventura, galbester@gmail.com

\begin{abstract}
Resumen
El tabaquismo es un problema de salud pública de primer orden. En los últimos años, las autoridades sanitarias de muchos países occidentales, entre ellos España, están haciendo serios esfuerzos para controlar este grave problema. No obstante, se percibe que el consumo de tabaco sigue siendo muy importante, alrededor del $20 \%$ en la población escolar de 14 a 16 años son fumadores. Además, alrededor del 50\% de los menores de 14 años viven en hogares donde se fuma. El consumo del tabaco durante el embarazo es una importante causa de morbimortalidad, tanto neonatal como a largo plazo. No existen estudios de suficiente calidad que evalúen la eficacia del consejo individual antitabaco en adolescentes. Los programas escolares y comunitarios tienen una eficacia reducida. Se recomienda realizar consejo antitabaco en padres y madres que asisten a la consulta de Pediatría. Es especialmente importante el consejo antitabaco en embarazadas. El consejo antitabaco en adolescentes debe ajustarse a su nivel de desarrollo y comprensión. Se debe insistir en las consecuencias a corto plazo y sobre aquellos aspectos más motivadores para los adolescentes. Se propone un folleto para administrar el consejo en adolescentes.
\end{abstract}

Palabras clave: Tabaquismo, Consejos.

\section{Abstract}

Smoking is one of the main Public Health problems. In many western countries, Spain among them. In the last few years in particular, Health authorities are working hard in order to get this important problem under control. In spite of this, it can be seen that tobacco use is still significant, thus in secondary school children, over $20 \%$ of adolescents aged 14-16 years are smokers. Moreover, around $50 \%$ of children younger than 14, have domestic exposure to environmental tobacco smoke. Smoking during pregnancy causes significant morbidity and mortality, not only in neonatal age but also throughout life. There are not enough studies of sufficient quality that evaluate the effectiveness of individual advice in adolescents. School and community programs have a limited efficacy. Anti-smoking counselling to parents in a Paediatric Primary Ca-

El autor declara no presentar posibles conflictos de intereses en relación con la preparación y publicación de este artículo. 
re setting is recommended. This is of especial importance in pregnant women. We must advise adolescents to stop smoking in a way that is adapted to their development and understanding capacity. We should stress the short-term consequences and the most important motivating aspects for teenagers. A special leaflet for adolescents giving anti-smoking counselling is suggested.

Key words: Smoking, Advice.

\section{Introducción}

El consumo de tabaco es la primera causa de muerte evitable en los países occidentales $^{1-2}$, por lo que se constituye en uno de nuestros principales problemas de salud pública. El tabaquismo es además un hábito que a menudo se adquiere en la adolescencia y se considera también un factor de riesgo para el consumo de marihuana y otras drogas.

Tabaquismo pasivo: el tabaco es la causa principal de contaminación ambiental presente en la vida de los niños que conlleva importantes problemas de salud. En un estudio realizado en 2007 en la ciudad de Zaragoza, más del $50 \%$ de los niños menores de 14 años vivían en hogares donde estaba presente el humo del tabaco ${ }^{3}$. Se calcula que esta exposición es equivalente al consumo de 4-10 cigarrillos por día o entre 60150 cigarrillos por año ${ }^{4}$. Los componentes químicos del humo secundario son más tóxicos que los de la corriente principal que se inhala al fumar. El riesgo es mayor para el feto y para los niños menores de 18 meses.
Riesgo durante la gestación: en el mencionado estudio realizado en Zaragoza $^{3}$, un $15,6 \%$ de las gestantes fumaron durante el embarazo y todas ellas continuaron fumando a lo largo del año siguiente. El tabaquismo materno durante la gestación representa riesgos específicos, ya que los niveles de cotinina fetal alcanzan el $90 \%$ de los de la ma$\mathrm{dre}^{4,5}$. El tabaquismo pasivo de la madre también es perjudicial para el feto y se estima que la exposición de una madre no fumadora al humo del tabaco puede suponer para el feto el equivalente del consumo hasta 4-5 cigarrillos por día $(\mathrm{Cpd})^{4}$. El consumo de tabaco durante la gestación se correlaciona con la presencia de importantes alteraciones placentarias demostradas mediante estudios anatomopatológicos, como por ejemplo la presencia de lesiones isquémicas e infartos placentarios, así como un aumento de los espacios intervellositarios. Todos estos cambios dan lugar a un menor peso del feto y a un mayor número de complicaciones obstétricas derivadas de la hipoxemia fetal que lleva a un aumento de riesgo de mutaciones celula- 
res, de embarazos ectópicos, de aumento de la mortalidad perinatal, alteraciones endocrinas y de la función respiratoria del niño.

Respecto al peso al nacimiento el tabaquismo exclusivamente paterno de al menos 20 Cpd se correlaciona con un peso al nacer menor estimado en $88 \mathrm{~g}$ inferior a la media. Cuando la madre es fumadora de la misma cantidad de cigarrillos, la media de peso al nacer se rebaja en $150 \mathrm{~g}^{4,5}$.

Síndrome de muerte súbita del lactante (SMSL): se han postulado diversas causas que llevan a un aumento de SMSL cuando la madre es fumadora, estas tienen que ver con anomalías a nivel del control respiratorio central y de la respuesta alterada a la hipoxemia durante el sueño REM inducida por la nicotina. También se han visto aumentos del número de apneas centrales ${ }^{4}$.

Tabaquismo y lactancia materna: se sabe que las madres fumadoras producen menos leche y con una menor cantidad de grasa que las no fumadoras. De esta manera la duración de la lactancia materna suele ser menor en las fumadoras. Por otra parte, se ha podido observar un cierto efecto protector de la lactancia materna sobre las infecciones respiratorias de vías bajas si esta se prolonga más allá de los 6 meses ${ }^{4}$.
Infecciones respiratorias de vías altas: son más frecuentes en los niños y niñas expuestos al humo del tabaco, especialmente si la madre es fumadora, y esto da lugar a que tienen un $70 \%$ más de infecciones respiratorias de vías altas, un mayor riesgo de otitis, faringitis y de infecciones ORL en general ${ }^{3-7}$.

Infecciones respiratorias de vías bajas: en el ya citado estudio realizado en Zaragoza se pudo comprobar que las infecciones respiratorias de vías bajas eran más frecuentes entre los expuestos al humo del tabaco de más de $20 \mathrm{Cpd}$ (OR [odds ratio] = 3,48; IC 95\%: 2,076) $)^{3}$. También aumentaban el número de episodios de sibilancias, bronquiolitis y asma bronquial. Se ha podido comprobar, asimismo, una peor función respiratoria a lo largo de la infancia, siendo mayor la repercusión si la exposición se produjo durante el embarazo $0^{3-7}$.

\section{Enfermedad meningocócica invasiva:} se ha descrito una mayor frecuencia de enfermedad meningocócica en niños expuestos al humo del tabaco, 5 veces más cuando la madre es fumadora, 4 cuando es el padre y 9 si ambos fuman ${ }^{4}$.

Ateromatosis: en estudios realizados sobre las arterias coronarias en niños, se ha comprobado la existencia de lesiones preateromatosas y su progresión a lesiones ateromatosas en niños expuestos 
al humo del tabaco con mucha mayor frecuencia que en los no expuestos ${ }^{4}$.

Otros trastornos: también se ha comprobado un mayor número de casos de neoplasias, TDAH y absentismo escolar en niños expuestos al humo del tabaco ${ }^{4}$.

\section{Tabaquismo activo}

La situación del consumo de tabaco en España en jóvenes de 14 a 18 años se analiza partiendo de los datos que aporta el Ministerio del Interior en su Plan Nacional de Lucha contra la Droga y con datos procedentes del año 2006 (tablas I y II).

\section{Consejo antitabaco en adolescentes}

Es una realidad, por otra parte, la escasa prevalencia del consejo breve en las consultas de Pediatría de Atención Primaria (AP); tampoco en las consultas de los dentistas es frecuente el consejo, a pesar de la alta tasa de frecuentación de ambas al cabo de un año. En un estudio realizado sobre la clientela de dentistas y pediatras de AP en EE. UU. se vio que alrededor de un $70 \%$ acudían a la consulta de uno u otro al cabo de un año. El estudio puso de manifiesto que el OR fue de un 1,81 (IC $95 \%$ : $1,61-2,04)$ para el grupo de fumadores habituales por parte del médico, y de 1,51 (IC 95\%: 1,34-1,72) 8.10 $^{\text {para el }}$ grupo de fumadores habituales por parte del dentista.

\section{Programas antitabaco: programas en la escuela}

En general, los programas pueden clasificarse en uno de estos grupos, basados cada uno en un modelo explicativo del consumo:

Tabla I. Consumo de tabaco. Encuesta bienal en años pares

\begin{tabular}{lcccc}
\hline & 2000 & 2002 & 2004 & 2006 \\
\hline Alguna vez & $61,8 \%$ & $59,8 \%$ & $60,4 \%$ & $46,1 \%$ \\
Últimos 30 días & $32,1 \%$ & $29,4 \%$ & $37,4 \%$ & $27,8 \%$ \\
\hline
\end{tabular}

Tomado de: Plan Nacional de Lucha contra la Droga, Ministerio del Interior, 2006.

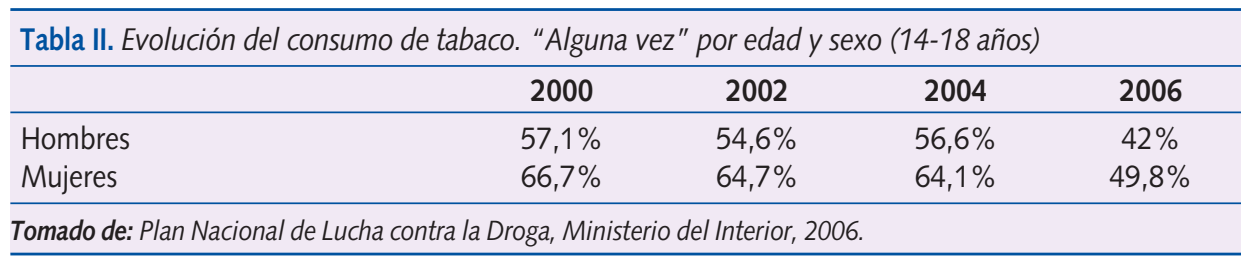


- Programas basados en la información. Los contenidos de estos programas se basan en aportar información sobre prevalencias de consumo de sustancias y riesgo de los mismos.

- Programas basados en la competencia social. Parten de la teoría del aprendizaje social de Bandura (Bandura, 1977), en la que se propone que el aprendizaje se produce por imitación y modelado de la conducta, más refuerzos positivos o negativos. A su vez, se ve influido por situaciones favorecedoras $y$ por determinadas habilidades sociales. Estos programas actúan mediante la instrucción, demostración, representación de papeles y refuerzos, así como en técnicas de afrontamiento del estrés y mejora de la autoestima.

- Programas basados en las influencias sociales. Se fundamentan en el modelo de Evans (Evans, 1976). En estos programas están presentes todos los elementos descritos en el apartado anterior, pero insisten en el uso de la educación normativa y en las técnicas específicas de resistencia ("capacidad para decir que no"), así como en la corrección de creencias que sobrees- timan la prevalencia del consumo de sustancias entre adolescentes y adultos ("no es tan normal...") y la presencia de comités contra el consumo o la enseñanza de técnicas conductuales específicas.

- Programas multimodales: combinan elementos de unos y otros $y$, además, incorporan actividades o programas dirigidos a padres, políticas comunitarias o medidas o iniciativas legislativas.

La evaluación de la eficacia es complicada por varias razones: lapso de tiempo largo entre implementación e inicio de posibles consumos, pérdidas de sujetos participantes que sesgan la evaluación, existencia de variables de confusión, unidad de evaluación a veces no muy clara.

Si analizamos una experiencia realizada en Zaragoza, donde se hizo una intervención comunitaria de base escolar, en la que se dio consejo antitabaco breve dirigido a escolares de $2 .^{\circ}, 3 .^{\circ}$ y $4 .^{\circ}$ de ESO en 15 centros de educación secundaria de esta ciudad observamos lo siguiente: se evaluaron 1.720 estudiantes entre los dos cursos 2006-07 y 2007-08 de los cuales el 51,95\% fueron hombres y el $48,5 \%$ mujeres. La edad media fue de 14,6 años (desviación estándar $[D E]=1,36$ ) y fueron fumadores 
el $19,7 \%$. Entre las mujeres fueron fumadoras el $24,7 \%$ frente al $18,3 \%$ de los hombres ( $p<0,0001)$. No hubo diferencias significativas para el tabaquismo según grupo de intervención o control. La progresión del tabaquismo creció un $8,8 \%$ en el grupo intervención frente a un $12 \%$ en el de control entre $2 .^{\circ}$ y $3 .^{\circ}$. Las diferencias fueron del $3 \%$ para el grupo intervención frente al $1,1 \%$ en el de control entre $3 .^{\circ}$ y $4 .^{\circ}$, no siendo estas diferencias estadísticamente significativas ${ }^{11}$.

Partiendo de los datos de la revisión de Thomas 1 en la biblioteca Cochrane, sobre los trabajos de mayor calidad, no existe evidencia de buena calidad para afirmar que los programas basados tan solo en información modifiquen el consumo de tabaco, aunque generen un mayor grado de conocimiento y actitudes negativas hacia el tabaco a corto plazo. Los programas basados en el modelo de influencias sociales parecen ser moderadamente eficaces, con diferencias de un $4 \%$ en prevalencia de consumo de tabaco en el último mes, frente a los basados tan solo en la información $y$, sobre todo, cuando se combinan con educación para resistir presiones'. Merece la pena destacar el proyecto Towards No Drug Abuse (TND) que consta de 9 sesiones: 3 sobre motivación, escucha, mitos y estereotipos; 3 sobre dependencia; y 3 sobre alternativas sin drogas. Se midieron consumos en una escala de 11 niveles de 0-100 cigarrillos por mes y se midió el CO espirado. Para el TND con 715 estudiantes de 18 escuelas en California se produjo una reducción en el consumo de un $27 \%$ al año del grupo liderado por educadores para la salud, respecto del grupo autoinstruido'.

\section{Programas antitabaco: programas comunitarios}

Son aquellos basados en la participación de la comunidad. Suelen incluir intervenciones en grandes poblaciones de diversos sectores, edades y clases sociales ${ }^{12-14}$. Es característico también que las intervenciones sean múltiples. La evaluación es difícil por la heterogeneidad de las intervenciones entre unas y otras comunidades, y el seguimiento y evaluación a medio y largo plazo es complicada.

En la revisión sistemática de Arblaster se identificaron 13 estudios metodológicamente adecuados ${ }^{12}$. Los estudios que comparaban intervención frente a no intervención o promoción estándar fueron 9, de ellos 2 informaron de reducciones en el consumo de tabaco en el grupo de intervención frente al de 
control. Ambos eran programas diseñados como estudios a largo plazo para la prevención de factores de riesgo cardiovascular. Se dirigían a toda la población e incluían actividades específicas dirigidas a escolares y en el marco escolar.

El estudio de Karelia del norte es un amplio estudio de prevención cardiovascular a lo largo de 8 años, que comenzó en 1972 y que incluyó una gran campaña mediática (Vartiainen, 1998). Durante $1978-80$ se añadieron dos componentes escolares diferentes al programa. Una de cada dos escuelas llevaban uno u otro programa entre las dos seleccionadas por provincia. El tercer par de escuelas de otra provincia siempre fue utilizado como control y no recibió intervención. Quince años después, cuando los componentes del estu- dio tenían 28 años, el consumo de tabaco fue un $22 \%$ más bajo en el grupo de intervención frente al de control. Por lo tanto, se puede concluir que algunos estudios bien diseñados y mantenidos en el tiempo muestran reducciones moderadas entre un $8-22 \%$ en el consumo de tabaco a medio y largo plazo ${ }^{12}$.

En resumen, los programas comunitarios parecen ofrecer un beneficio pequeño a favor de los grupos de intervención en la prevención de tabaquismo.

Las recomendaciones sobre consejo antitabaco breve que se proponen para utilizar en las consultas de Pediatría o Enfermería de AP se recogen en la tabla III. La fuerza de la recomendación de cada una de las intervenciones figura en la tabla IV. Se puede utilizar el test de Fageström breve para valorar la depen-

Tabla III. Recomendaciones de PAPPS/Prev/nfad ${ }^{14}$

- Realizar anamnesis de consumo y consejo breve antitabaco en padres y madres al menos una vez cada 2 años y especialmente en:

- Grupos socialmente desfavorecidos.

- Si hay patología relacionada con el tabaco.

- Gestantes.

- Derivar a consulta antitabaco si ello es posible en casos indicados.

- Realizar anamnesis en adolescentes, bien de forma programada en las revisiones sistemáticas, bien de forma oportunista en las consultas por patología relacionada, al menos una vez al año.

- Clasificar a los fumadores según su nivel de consumo y actitudes de cambio.

- Realizar test de Fagërstrom para valorar la dependencia y de Richmond de motivación al cambio.

- Realizar consejo breve antitabaco según metodología propuesta.

- Derivar para terapia cognitivo conductual, si hay recursos para ello, a todo fumador regular con elevada motivación para el cambio.

- Participar, si ello es posible, en programas y actividades comunitarias y escolares de prevención del tabaquismo. 
Tabla IV. Programas antitabaco. Calidad de la evidencia y fuerza de la recomendación

\begin{tabular}{|c|c|c|c|}
\hline Actividad & Población & Resultados esperados & $\begin{array}{l}\text { Fuerza de la } \\
\text { recomendación }\end{array}$ \\
\hline Programas escolares & 6-18 años & $\begin{array}{l}\text { Aumentan los conocimientos y } \\
\text { mejoran las actitudes frente al tabaco }\end{array}$ & B \\
\hline Programas escolares & 6-18 años & Disminuye el consumo de tabaco & I \\
\hline Programas comunitarios & Adolescentes & Disminuye el consumo de tabaco & I \\
\hline $\begin{array}{l}\text { Programas basados en los } \\
\text { medios de comunicación }\end{array}$ & Adolescentes & Disminuye el consumo de tabaco & I \\
\hline $\begin{array}{l}\text { Consulta breve } \\
\text { en Atención Primaria }\end{array}$ & Adultos & Disminuye el consumo de tabaco & A \\
\hline $\begin{array}{l}\text { Consulta breve } \\
\text { en Atención Primaria }\end{array}$ & Gestantes & Disminuye el consumo de tabaco & I \\
\hline $\begin{array}{l}\text { Consulta breve } \\
\text { en Atención Primaria }\end{array}$ & Adolescentes & Disminuye el consumo de tabaco & I \\
\hline
\end{tabular}

Tomado de: Canadian Preventive Services Task Force (CPSTF).

Tabla V. Test de Fagërstrom (breve)

¿Cuánto tiempo pasa después

de despertarse en fumar su primer

cigarrillo?

- $<5$ minutos: 3

- 6-30 minutos: 2

- 31-60 minutos: 1

- >60 minutos: 0
¿Cuántos cigarrillos fuma por día?

- > 30: 3

- 21-30: 2

- 11-20: 1

- $<11: 0$

Puntuación:

0 a 2: dependencia baja

3 a 4: dependencia moderada

5 a 6: dependencia alta dencia (tabla V). Finalmente, la figura 1 recoge un folleto de apoyo para el consejo breve en adolescentes.

\section{Estrategias de búsqueda}

Medline, Embase, Proquest, Cochrane Library, National Clearinghouse, Tripdatabase, Google:

("Tobacco" $[\mathrm{MeSH}] \mathrm{OR}$ "Tobacco Use Cessation"[MeSH]) AND "Smoking" [MeSH] AND ("Adolescent" [MeSH] OR
"Adolescent Health Services" [MeSH] OR "Adolescent Behavior" [MeSH] OR "Adolescent Development"[MeSH] OR "Adolescent Psychology"[MeSH] OR "Adolescent ("Counseling/education" [MAJR] OR "Counseling/utilization" [MAJR]) AND "pregnancy"[MESH] AND "Substance-Related Disorders/ prevention and control"[MAJR] AND "smoking/prevention and control" [MAJR]). 
Figura 1. Folleto para el consejo antitabaco en adolescentes. Grupo Aragonés de Investigación en Atención Primaria.

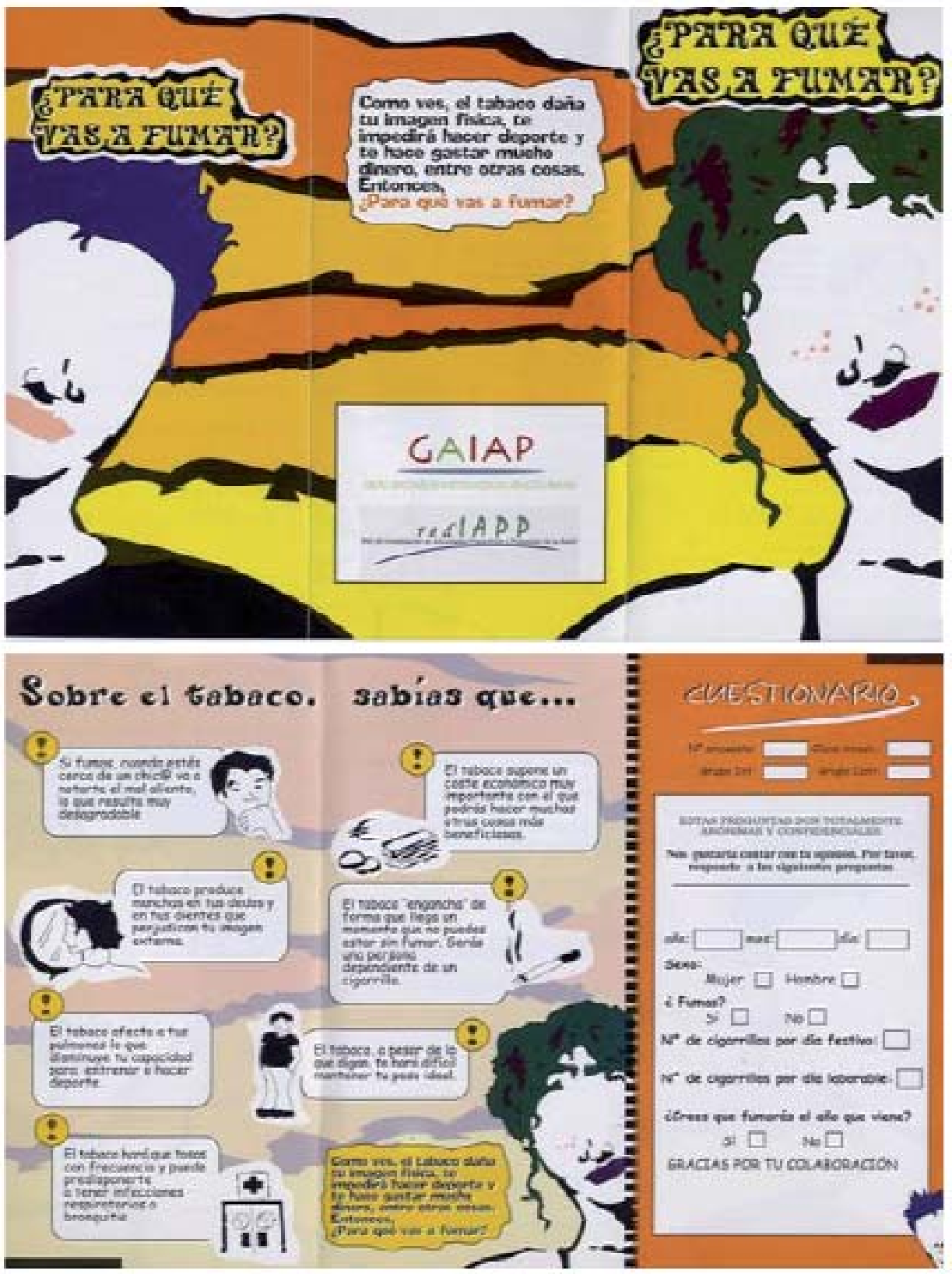


("Mass media"[MESH] AND "mass media/utilization"[MAJR] AND ("Substance-Related Disorders/epidemiology" [MESH] OR "Substance-Related Disorders/legislation and jurisprudence" [MESH] OR "Substance-Related Disorders/prevention and control" [MESH] OR "Substance-Related Disorders/trends" [MESH]) AND "drinking" [MESH] AND "Alcoholism/prevention and control" [MESH] AND ("smoking/prevention and control" [MESH] OR "smoking/trends" [MESH]) AND "tobacco"[MESH] AND "Adolescent"[MAJR] Medicine"[MeSH]).
Adolescent And Smoking Or Tobacco And Counselling.

Adolescent And Smoking Or Tobacco And Nicotine.

Adolescent And Smoking Or Tobacco And Bupropion.

Adolescent And Smoking Or Tobacco And Expired COearch.

(("Tobacco Smoke Pollution" [Mesh] OR "Tobacco Smoke Pollution/adverse effects" [Mesh])) AND "Tobacco Smoke Pollution/adverse effects"[Mesh] Limits: Meta-Analysis, Practice Guideline, All Child: 0-18 years.

\section{Bibliografía}

1. Thomas R. Programas escolares para la prevención del tabaquismo. En: Biblioteca Cochrane Plus 2005, n. ${ }^{\circ}$ 2. Oxford Update Software Ltd. Disponible en www.update.software.com (traducido de The Cochrane Library. 2005; Issue 2. Chichester UK John Wiley \& Sons Ltd) [consultado el 17/07/2002].

2. Alonso de la Iglesia $B$, Castañal $X$, Domínguez Gandal F. Tabaquismo, algo más que una epidemia. En: Abordaje del tabaquismo en Atención Primaria. Curso on-line. Barcelona: semFYC ed.; 2005.

3. Marco Tejero A, Pérez Trullén A, Córdoba García R, García Sánchez N, Cabañas Bravo MJ. La exposición al humo de tabaco en el hogar aumenta la frecuentación por patología respiratoria en la infancia. An Pediatr (Barc). 2007;66(5):475-80.

4. Córdoba García R, García Sánchez N, Suárez de Vergara RG, Galván Fernández C. Exposición al humo de tabaco en la infancia. An Pediatr (Barc). 2007:67(2):101-3.

5. Jedrychowski W, Galas A, Pac A, Flak E, Camman $D$, Rauh V, et al. Prenatal ambient air exposure to polycyclic aromatic hydrocarbons and the occurrence of respiratory symptoms over the first year of life. European J Epidemiology. 2005;20:775-82.

6. Eisner $M$, Forastiere F. Passive smoking, Lung Function and Public Health. Am J Respir Crit Care Med. 2006;173(11):1184.

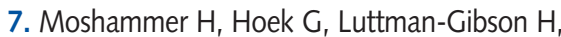
Neuberger N, Tamenuga A, Gehring V, et al. Parental Smoking and Lung Function in Children. An International Study. Am J Respir Crit Care. 2006; 173(11):1255-63.

8. Secker-Walker RH, Warden $\mathrm{JH}$, Holland RR, Flyn BS, Detsky S. A mass media programme to prevent smoking among adolescence: cost and cost-effectiveness. Tobacco control. 1997;6(3):207-12.

9. Pérez-Stable $E$, Juárez Reyes $M$, Kaplan $C$, Fuentes-Afflick $E$, Gildengorin V, Millstein V. 
Counseling smoking parents of young children. Comparison of Pediatricians and Familiy Physicians. Arch Pediatr Adolesc Med. 2001;155(1):2532.

10. Kaplan C, Pérez-Stable EJ, Fuentes-Afflick E, Gildengorin V, Millstein S, Juárez-Reyes M. Smoking cessation counselling with youth patients. The practices of Family Physicians and Pediatricians. Arch Pediatr Adolesc Med. 2004;158(1):83-92.

11. Galbe J, Traver P, Galve Z, Aliaga Y, Navarra B, Duplá $M, y$ cols. Estudio de consejo breve para la prevención del tabaquismo en escolares de $2 .^{\circ}$ a 4. ${ }^{\circ}$ de ESO de Zaragoza. Rev Pediatr Aten Primaria. 2009;11:49-63.
12. Arblaster N, Sowden A. Community interventions for preventing smoking in young people. Cochrane Rev. 2002;2:1-30.

13. Morales López R, González Rosales E, Asensio López $\mathrm{S}$. Intervenciones comunitarias en tabaquismo. En: Abordaje del tabaquismo en Atención Primaria. Curso on-line. Barcelona: semFYC ed.; 2005.

14. Galbe J y Grupo Previnfad. Prevención del tabaquismo en la adolescencia [consultado el 25/08/2009]. Disponible en www.aepap.org/pre vinfad/pdfs/previnfad_tabaco.pdf 